\title{
Status of Vitamin D-25 Hydroxy Vitamin D-25 (OH) in Patients With Multiple Myeloma
}

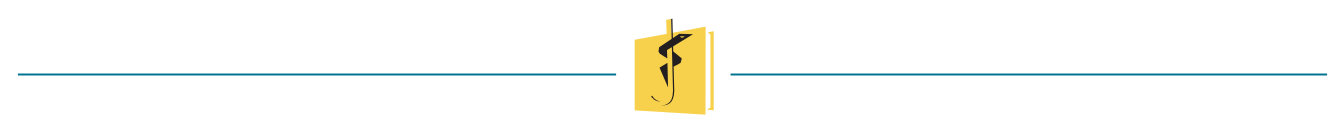

Donaryn V. Pasamonte', Flordeluna Z. Mesina M.D. ${ }^{2}$

\section{ABSTRACT}

Introduction Multiple myeloma (MM) causes generalized bone loss leading to lytic bone lesions and pathologic fractures. The increased osteoclast activity and reduced osteoblast function favors bone resorption and decreased bone formation. Vitamin $D$ is vital in regulating calcium homeostasis and osteoclast-mediated bone resorption. Deficiency of Vitamin D among MM patients may complicate bone mineralization problems and fractures.

\section{Objective}

General Objective To determine the status of Vitamin $D$ in patients with multiple myeloma

Specific Objective To determine the levels of Vitamin D, intact parathyroid hormone and ionized calcium among MM patients

Methodology This is a prospective, cross-sectional study which included patients who were 18 years old and above, male or female, diagnosed with MM at the University of Santo Tomas Hospital, with or without treatment. Excluded in the study were those with Vitamin D and calcium supplementation. Eligible subjects were extracted blood for Vitamin D

Donaryn Pasamonte

docdonaryn@gmail.com

1 University of Santo Tomas Hospital, Internal Medicine Department, Section of Clinical Hematology

2 University of Santo Tomas, Department of Medicine and Pharmacology assay, intact parathyroid hormone and ionized calcium.

Results A total of 22 patients with $M M$ were included in the study. Sixteen patients (72.7\%) had hypovitaminosis D. Among these sixteen patients, seven $(31.8 \%$ ) had Vitamin D deficiency (Vitamin D levels $<20 \mathrm{ng} / \mathrm{mL}$ [50 nmol/L]) and nine $(40.9 \%)$ had Vitamin D insufficiency (levels of 21-29 ng/ $\mathrm{mL}[52.5-72.5 \mathrm{nmol} / \mathrm{L}])$. Only $6(27.3 \%)$ of them were found to have normal serum Vitamin $D$ (levels of $>29 \mathrm{ng} / \mathrm{mL}[>72.5 \mathrm{nmol} / \mathrm{L}])$. The mean age $(p=0.069)$, intact PTH $(p=0.062)$ and ionized calcium $(p=0.188)$ of the three groups of patients did not differ.

Conclusion This study found a high incidence of Vitamin $D$ deficiency among $M M$ patients seen at the University of Santo Tomas Hospital. Vitamin D deficiency was independent of age, intact PTH and ionized calcium. It was more common in male subjects. Patients with hypovitaminosis D are at risk of having secondary hyperparathyroidism.

Recommendation Vitamin D status should be determined among patients with MM. Early recognition and treatment of hypovitaminosis $D$ will prevent the risk of having secondary hyperparathyroidism that can complicate skeletal-related events.

\section{INTRODUCTION}

Multiple myeloma (MM) is an abnormal proliferation of plasma cells in the bone marrow. The clinical manifestation is variable, which can range from 
being asymptomatic to multi-organ involvement. This plasma cell malignancy affects mostly the kidneys and the skeletal system causing renal insufficiency, hypercalcemia, hyperviscosity and bone disease. Skeletal pathology in $M M$ is characterized by generalized bone loss which can present as bone pain, lytic bone lesions or pathologic fractures. These result from increased osteoclast activity and reduced osteoblast function $[1,2,3]$ causing increased bone resorption and decreased bone formation.

Bone health, in general, is maintained by the balance of the following elements: calcium, Vitamin D and parathyroid hormone. Vitamin D is the most important regulator of calcium homeostasis and osteoclast-mediated bone resorption. Hypovitaminosis D adversely affects calcium metabolism, osteoblastic activity, matrix ossification, bone remodeling and bone density [4]. Other consequences of Vitamin $D$ deficiency are secondary hyperparathyroidism and bone loss leading to osteoporosis and fractures, mineralization defects, and muscle weakness causing falls and subsequently leading to more fractures [5]. Incidence of Vitamin D deficiency and insufficiency among MM patients is high [6]. This deficiency can cause generalized musculoskeletal pain and increase risks of fall [7], and hence may create further debilitating problems in this group of patients. In the Philippines, there is no data on the incidence or prevalence of Vitamin D deficiency among MM patients. This study was conducted to determine the status of Vitamin D among this subset of patients from University of Santo Tomas Hospital (USTH).

\section{MATERIALS AND METHODS}

This is a prospective, cross-sectional study which included patients who were 18 years old and above, male or female, diagnosed with MM at the USTH, with or without treatment. Excluded in the study were those with Vitamin D and calcium supplementation. Written consent was signed by all subjects after thoroughly explaining the study to them. After signing a USTH-Institutional Review Board approved informed consent, subjects were instructed to fast for 8-10 hours prior to blood extraction. The following blood tests were requested: Vitamin D assay, intact parathyroid hormone and ionized calcium. The laboratory tests were done at the USTH laboratory. Specimen acquisition and handling were in accordance with the strict laboratory practice of the USTH clinical pathology laboratory. Results were given to the patient, attending physician and investigator for data collection, recording and analysis.

\section{STATISTICAL ANALYSES}

MM patients' mean and range of vitamin $D$ are presented in Table 1. Means and standard deviations summarized the data in quantitative form, such as age, intact parathyroid hormone (PTH), ionized calcium, albumin, globulin and total protein (TP). Counts

Table 1. Demographic and serum profile of multiple myeloma patients

\begin{tabular}{llllll}
\hline \multicolumn{5}{c}{ Vitamin D level } \\
\hline & ALL & Normal & Insufficient & Deficient & p-value \\
Number of Patients & 22 & 6 & 9 & 7 & 0.659 \\
Age & $61.36 \pm 10.06$ & $64.17 \pm 5.74$ & $61.44 \pm 10.70$ & $58.86 \pm 10.93$ & 0.048 \\
Sex (M/F) & $10 / 12$ & $2 / 4$ & $7 / 2$ & $1 / 6$ & 1.000 \\
ECOG score: 0 & $8(36.4 \%)$ & $2(33.3 \%)$ & $4(44.4 \%)$ & $2(28.6 \%)$ & $2(28.6 \%)$ \\
& $5(22.7 \%)$ & $1(16.7 \%)$ & $2(22.2 \%)$ & $3(42.9 \%)$ & \\
Intact PTH & $9(40.9 \%)$ & $3(50 \%)$ & $3(33.3 \%)$ & $29.36 \pm 16.79$ & 0.062 \\
Normal/Decreased & $26.58 \pm 14.79$ & $14.86 \pm 5.74$ & $32.23 \pm 14.01$ & $5 / 2$ & 0.011 \\
lonized Calcium & $16 / 6$ & $2 / 4$ & $9 / 0$ & $1.26 \pm 0.17$ & 0.188 \\
Albumin & $1.35 \pm 0.29$ & $1.24 \pm 0.03$ & $1.48 \pm 0.41$ & $4.15 \pm 0.38$ & 0.048 \\
Globulin & $3.74 \pm 0.62$ & $3.76 \pm 0.57$ & $3.41 \pm 0.65$ & $2.93 \pm 0.87$ & 0.269 \\
TP & $3.82 \pm 2.06$ & $4.99 \pm 2.80$ & $3.74 \pm 2.05$ & $7.23 \pm 1.40$ & 0.403 \\
\hline
\end{tabular}

Values expressed as counts (\%), or mean \pm SD.

M, male; F, female; ECOG, Eastern Cooperative Oncology Group; PTH, parathyroid hormone; TP, total protein 
and percentages summarized the data in qualitative form, such as gender, Eastern Cooperative Oncology Group (ECOG) score, and decreased/ normal level of PTH. Single-factor analysis of variance with Tukey's HSD determined if there were significant differences in the mean age, PTH, ionized calcium, albumin, globulin and TP of patients with normal, insufficient and deficient Vitamin D. Fisher's exact test determined if there were significant association of levels of Vitamin D with gender, ECOG score and decreased/normal level of PTH. Pearson correlation identified the correlation of PTH, ionized calcium, albumin, globulin and TP. P-values less than 0.05 are considered significant. All statistical tests are performed using $\mathrm{R}$ ver 3.2.2.

\section{RESULTS}

A total of 22 patients with $M M$ were included in the study. The mean age $(p=0.069)$, PTH $(p=0.062)$, ionized calcium $(p=0.188)$, globulin $(p=0.269)$ and TP $(p=0.403)$ of the three groups of patients did not differ. Similarly, ECOG scores are not associated with the levels of Vitamin D of patients ( $p=1.000)$.

Meanwhile, it was evident that gender is associated $(p=0.048)$ with the level of Vitamin $D$, indicating that males are 16.2 times $[95 \% \mathrm{Cl}: 1.1$ to 1008.4] more likely to have vitamin D deficiency as compared to those with insufficient Vitamin D. More male $M M$ patients are suffering from hypovitaminosis D (80\%; deficient and insufficient patients combined), compared to their female counterparts (66.7\%).

The levels of PTH are significantly associated $(p=0.011)$ with their levels of Vitamin $D$, which implies that patients with insufficient levels of Vitamin $D$ are $97 \%$ less likely $[O R=0.03,95 \% \mathrm{Cl}: 0.00$ to 0.74 ] to have decreased PTH compared to those with normal levels of Vitamin D.

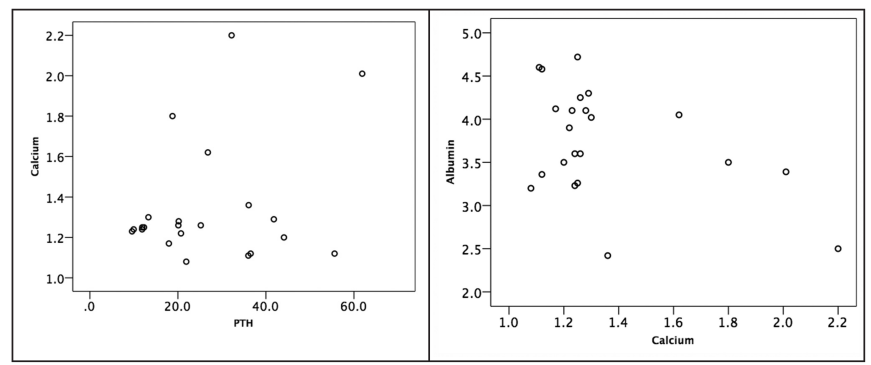

PTH and calcium $(r=0.259, p=0.244)$ are not significantly correlated. Relationship of calcium and albumin is also not significant $(r=-0.338, p=0.185)$.
Of the 22 patients included in the study, only 6 $(27.3 \%)$ of them were found to have normal serum Vitamin D, while the remaining $16(72.7 \%)$ had Vitamin D below $29 \mathrm{ng} / \mathrm{mL}$ (the normal reference: $>29 \mathrm{ng} / \mathrm{mL}$ [>72.5 nmol/L]). Seven (31.8\%) patients had Vitamin D deficiency (levels $<20 \mathrm{ng} / \mathrm{mL}$ [50 nmol/L]) and nine (40.9\%) were categorized as having insufficient Vitamin D (levels of $21-29 \mathrm{ng} / \mathrm{mL}$ [52.5-72.5 nmol/L]).

\section{DISCUSSION}

MM remains to be an incurable plasma cell malignancy despite the advent of newer pharmacological treatment modalities [8]. Renal insufficiency and osteolytic bone disorders are among the systemic complications of this disease. Hypercalcemia results from increased bone resorption leading to skeletal-related events like pathologic fractures. The cornerstone in the treatment of high calcium levels among MM patients includes hydration and initiation of bisphosphonates [9].

Vitamin D has a very important role in skeletal physiology as it is required for bone mineralization and formation [10]. The active metabolite 1, $25(\mathrm{OH}) 2 \mathrm{D}$ stimulates the absorption of calcium from the intestine [5]. The Vitamin D precursors, D2 and D3 are metabolized to 25-hydroxyvitamin D $[25(\mathrm{OH}) \mathrm{D}]$ in the liver and serum $25(\mathrm{OH}) \mathrm{D}$ is used to assess Vitamin D status [1 1]. Vitamin D deficiency is defined as a $25(\mathrm{OH}) \mathrm{D}$ below $20 \mathrm{ng} / \mathrm{mL}(50 \mathrm{nmo}-$ $\mathrm{I} / \mathrm{L})$ and Vitamin $D$ insufficiency as a $25(\mathrm{OH}) \mathrm{D}$ of 21-29 ng/mL (52.5-72.5 nmol/L) [4].

The major cause of Vitamin D deficiency is lack of sun exposure. It is more prevalent in places with winter season making ultraviolet light (UV) unavailable for the skin to manufacture Vitamin D [1 1]. In tropical countries like the Philippines, hypovitaminosis $D$ was noted to be common among office workers who stay indoors most of the day. The habitual wearing of long-sleeved clothing and slacks or pants, using too much sunscreen and lack of Vitamin D-rich foods in the diet are all contributory to having Vitamin D deficiency [12].

Studies have proven that there is a high incidence of Vitamin D deficiency among MM patients $[6,7]$. More importantly, $\mathrm{Ng}$ et al. [13] stated that hypovitaminosis $D$ may portend poorer disease outcomes in patients with MM. A study done by Aribi et al. [14] in Algeria concluded that Vitamin D could be 
considered as an effective prognostic factor and treatment of $M M$, especially in patients with monoclonal lgG kappa immunoglobulin. One case study reported a 63-year-old MM patient who had Vitamin $D$ deficiency and highlighted that Vitamin $D$ deficiency can cause generalized musculoskeletal pain and increase risks of fall [8]. Maier et al. [15] also found out that there is widespread and alarming rate of hypovitaminosis $D$ among patients with metastatic bone disease and MM. In that same study, it was concluded that it is important to assess Vitamin $D$ levels among these subsets of patients.

This study confirmed that Vitamin D deficiency is prevalent among MM patients seen at the USTH. Of the 22 patients included in the study, only $6(27.3 \%)$ of them were found to have normal serum Vitamin $\mathrm{D}$, while the remaining $16(72.7 \%)$ had Vitamin D below $29 \mathrm{ng} / \mathrm{mL}$ (the normal reference: $>29 \mathrm{ng} / \mathrm{mL}$ [>72.5 nmol/L]). Seven (31.8\%) patients have Vitamin D deficiency (levels $<20 \mathrm{ng} / \mathrm{mL}$ [50 nmol/L]) and nine $(40.9 \%)$ were categorized as having insufficient Vitamin D (levels of 21-29 ng/mL [52.5-72.5 nmoI/L]). Table 1 details the number of patients belonging to the sufficient, insufficient and deficient groups.

Table 2 compiles the demographics and serum profile of $M M$ patients included in the study. The mean age $(p=0.069)$, PTH $(p=0.062)$, ionized calcium $(p=0.188)$, globulin $(p=0.269)$ and TP $(p=0.403)$ of the three groups of patients did not differ. However, more male $M M$ patients are suffering from hypovitaminosis D $180 \%$; deficient and insufficient patients combined) compared to their female counterparts $(66.7 \%)$. This individual variability of $25(\mathrm{OH}) \mathrm{D}$ levels may be due to differences in sunshine exposure, clothing style, skin pigmentation and thickness, age and weight [4].

Serum calcium level regulates PTH secretion via negative feedback through the parathyroid calcium-sensing receptor (CASR) [16]. Decreased calcium levels stimulate PTH release. However, this PTH and calcium relationship was not seen in this study

Table 2. Vitamin D level of patients

\begin{tabular}{lll}
\hline Vitamin D level & $\mathbf{n}$ & Mean (Range) \\
\hline Normal & 6 & $42.85(34.71-58.01)$ \\
Insufficient & 9 & $24.08(20.10-28.11)$ \\
Deficient & 7 & $16.05(11.64-19.29)$ \\
\hline
\end{tabular}

$n$ : number of patients $(r=0.259, p=0.244)$. An elevated PTH value with normal serum calcium may not necessarily indicate primary hyperparathyroidism. It is possible that the elevation in PTH is due to secondary causes, the most likely being Vitamin D deficiency [16]. The levels of PTH are significantly associated $(p=0.011)$ with their levels of Vitamin $D$, which implies that patients with insufficient levels of Vitamin D are $97 \%$ less likely $[\mathrm{OR}=0.03,95 \% \mathrm{Cl}: 0.00$ to 0.74$]$ to have decreased PTH compared to those with normal levels of Vitamin D. Patients with hypovitaminosis D are therefore more prone to having secondary hyperparathyroidism, which can further cause mineralization problems and bone loss, making $\mathrm{MM}$ patients more susceptible to pathologic fractures.

There was no significant correlation between Vitamin D status and performance status (ECOG score). This could be due to the differences in skeletal-related events experienced by the subjects. Some presented with bone pain without fractures, while those with fractures had varied sites of skeletal involvement.

\section{CONCLUSION AND RECOMMENDATIONS}

This study proved that there is a high prevalence of Vitamin D deficiency among MM which matched previous reports by Badros et al. and Laroche et al $[17,18]$. Our data showed that more male MM patients are suffering from hypovitaminosis D $180 \%$; deficient and insufficient patients combined), compared to their female counterparts $(66.7 \%)$. These Vitamin D-deficient subjects are more likely to have secondary hyperparathyroidism. It is recommended that MM patients should be screened for possible hypovitaminosis $D$ and prompt correction is necessary to avoid further bone loss. The International Myeloma Working Group recommends routine supplementation with calcium and Vitamin D among MM patients receiving bisphosphonate therapy [8]. In a study done by Hospital for Special Surgery in New York, they demonstrated that maintaining a circulating Vitamin D level above $33 \mathrm{ng} / \mathrm{mL}$ is associated with more favorable outcome with bisphosphonate therapy among patients with osteoporosis and low mineral density [19]. We recommend that a study be conducted among MM patients to determine if maintaining such a level will also optimize the effects of bisphosphonate in this subset of patients. 


\section{REFERENCES:}

1. Kyle R, Gertz M, Witzig T, Lust J, et. al. Review of 1027 patients with newly diagnosed multiple myeloma. Mayo Clinic Proceedings. 2003;78(2):21-3.

2. Silbermann R, Roodman D. Myeloma bone disease: Pathophysiology and management. Journal of Bone Oncology. 2013Jun;2(2):59-69.

3. Raje N, Roodman GD. Advances in the biology and treatment of bone disease in multiple myeloma. Clinical Cancer Research. 2011 Mar 15;17(6):1278-86.

4. Christodoulou S, Goula T, Ververidis A, Drosos G. Vitamin $D$ and bone disease. BioMed Research International. 2013;396541. Epub 2013/03/20. doi: 10.1155/2013/396541 PMID: 23509720.

5. Lips P, Van Schoor NM. The effect of vitamin D on bone and osteoporosis. Best Practice \& Research Clinical Endocrinology \& Metabolism. 2011 Aug 1;25(4):585-91.

6. Lauter B, Schmidt-Wolf IG. Prevalence, supplementation, and impact of vitamin $D$ deficiency in multiple myeloma patients. Cancer Investigation. 2015 Nov 26;33(10):505-9.

7. Clement Z, Ashford M, Sivakumaran S. Vitamin D deficiency in a man with multiple myeloma. North American Journal of Medical Sciences. 2011:469-71.

8. Terpos E, Morgan G, Dimopoulos MA, Drake MT, Lentzsch $S$, Raje $N$, et al. International Myeloma Working Group recommendations for the treatment of multiple myelomarelated bone disease. Journal of Clinical Oncology. 2013 Jun 20;31(18):2347.

9. Rajkumar SV, Kyle RA, Connor RF. Clinical features, laboratory manifestations, and diagnosis of multiple myeloma. UpToDate. 2011 Jun.

10. Eisman JA, Bouillon R. Vitamin D: direct effects of vitamin $D$ metabolites on bone: lessons from genetically modified mice. BoneKEy Reports. 2014 Feb 5;3.

11. Winzenberg T, van der Mei I, Mason RS, Nowson C, Jones G. Vitamin D: and the musculoskeletal health of older adults. Australian Family Physician. 2012 Mar;41(3):92.

12. Orosa R. 3 out of 5 Filipinos suffer from Vit-D deficiency. The Philippine Star. 2014 May 13;

13. Ng AC, Kumar SK, Rajkumar SV, Drake MT. Impact of vitamin $D$ deficiency on the clinical presentation and prognosis of patients with newly diagnosed multiple myeloma. American Journal of Hematology. 2009;84(7):397-400.

14. Aribi M, Senouci-Bereksi S, Haddouche M, Mesli N. Vitamin $D$ intake and light chain-lgG monoclonal antibodies in patients with multiple myeloma. $2012 \mathrm{Apr}$;

15. Maier GS, Seeger JB, Horas K, Roth KE, Kurth AA, Maus $U$. The prevalence of vitamin $D$ deficiency in patients with vertebral fragility fractures. The Bone \& Joint Journal. 2015;97-B(1):89-93.

16. Parathyroid Hormone (PTH), Swerum [Internet]. Mayo Clinic Laboratories. Available from: https://www.mayocliniclabs.com/test-catalog/Clinical and Interpretive/28379

17. Badros A, Goloubeva O, Terpos E, Milliron T, Baer MR, Streeten E. Prevalence and significance of vitamin D deficiency in multiple myeloma patients. British Journal of Haematology. 2008; 142(3):492-4.

18. Laroche $M$, Lemaire $O$, Attal M. Vitamin D deficiency does not alter biochemical markers of bone metabolism before or after autograft in patients with multiple myeloma. European Journal of Haematology. 2010;85:65-7.

19. Fisher P, Shieh A, Carmel A. Study finds high levels of Vitamin $D$ needed for bone density drugs to work. Hospital for Special Surgery. 2011. Available from: https://www.hss. edu/newsroom_high-vitamin-bone-drugs-work.asp

\footnotetext{
(c) (i) Open Access This article is licensed under a Creative Commons Attribution 4.0 International License, which permits use, sharing, adaptation, distribution and reproduction in any medium or format, as long as you give appropriate credit to the original author(s) and the source, provide a link to the Creative Commons license, and indicate if changes were made. The images or other third party material in this article are included in the article's Creative Commons license, unless indicated otherwise in a credit line to the material. If material is not included in the article's Creative Commons license and your intended use is not permitted by statutory regulation or exceeds the permitted use, you will need to obtain permission directly from the copyright holder. To view a copy of this license, visit http://creativecommons.org/licenses/ by/4.0/.
} 